\title{
Measuring the completeness of death registration in 2844 Chinese counties in 2018
}

Xinying Zeng ${ }^{1}$, Tim Adair ${ }^{2}$, Lijun Wang ${ }^{3}$, Peng Yin ${ }^{3}$, Jinlei Qi ${ }^{3}$, Yunning Liư ${ }^{3}$, Jiangmei Liư ${ }^{3}$, Alan D. Lopez ${ }^{2}$ and Maigeng Zhou $^{3^{*}}$

\begin{abstract}
Background: Death registration completeness has never been assessed at the county level in China. Such analyses would provide critical intelligence to monitor the performance of the vital registration system and yield adjustment factors to correct death registration data, thereby increasing their policy utility.

Methods: We estimated the completeness of death registration for 31 provinces and 2844 counties of China in 2018 based on death data from the China Cause of Death Reporting System (CDRS) by using the empirical completeness method. We computed the root mean square difference (RMSD) of county-level completeness compared with provincial-level completeness to study intra-provincial variations. A two-level (province and county) logistic regression model was fitted to explore the association between county-level registration completeness and a set of covariates reflecting socioeconomic status, healthcare quality, and specific strategies and regulations designed to improve registration.

Results: In 2018, the overall death registration completeness for the CDRS in China was 74.2\% (95\% uncertainty interval [UI] 66.2-80.4), with very little difference for males and females. Geographical differences in completeness were higher across counties than across provinces. The county-level completeness ranged from 2.4\% (95\% UI 1.05.0\%) in Burang County, Tibet, to 100.0\% (95\% UI 99.9-100.0\%) in Guandu District, Yunnan. The coastal provinces of Jiangsu, Guangdong, and Fujian, with higher overall completeness, contained counties with low completeness; conversely, the underdeveloped provinces of Guangxi and Guizhou, with lower overall completeness, included some counties with high completeness. GDP, education, population density, minority population, healthcare access, and registration strategies were important drivers of the geographical differences in registration completeness.

Conclusions: There are marked inequalities in registration completeness at the county level and within provinces in China. The socioeconomic condition, the implementation of specific registration-enhancing initiatives, and the availability and quality of medical care were the primary drivers of the observed geographical variation. A more strategic approach, with more research, is required to identify the main reasons for death under-reporting, especially in the poorer performing counties, to guide remedial action.
\end{abstract}

Keywords: Completeness of death registration, County level, China

\footnotetext{
* Correspondence: maigengzhou@126.com

${ }^{3}$ National Center for Chronic and Noncommunicable Disease Control and Prevention, Chinese Center for Disease Control and Prevention, 27\# Nanwei Road, Xicheng District, Beijing 100050, China

Full list of author information is available at the end of the article
}

(c) The Author(s). 2020 Open Access This article is licensed under a Creative Commons Attribution 4.0 International License, which permits use, sharing, adaptation, distribution and reproduction in any medium or format, as long as you give appropriate credit to the original author(s) and the source, provide a link to the Creative Commons licence, and indicate if changes were made. The images or other third party material in this article are included in the article's Creative Commons licence, unless indicated otherwise in a credit line to the material. If material is not included in the article's Creative Commons licence and your intended use is not permitted by statutory regulation or exceeds the permitted use, you will need to obtain permission directly from the copyright holder. To view a copy of this licence, visit http://creativecommons.org/licenses/by/4.0/. The Creative Commons Public Domain Dedication waiver (http://creativecommons.org/publicdomain/zero/1.0/) applies to the data made available in this article, unless otherwise stated in a credit line to the data. 


\section{Background}

Reliable and timely information on mortality patterns and levels for national and subnational populations is fundamental for informing the development, implementation, and evaluation of health policy [1]. In China, such intelligence is particularly critical given the large population spread over a vast area with rapid epidemiological transition bringing numerous challenges, including an aging population and a significant burden of noncommunicable diseases.

Prior to 2013, the essential data on the causes of death in China were provided by two sample-based mortality surveillance systems, the nationally representative Disease Surveillance Points (DSP) system of the Chinese Center for Disease Control and Prevention (CDC), and the vital registration (VR) system of the Chinese Ministry of Health $(\mathrm{MOH})$ [2]. The DSP system was initially established in 1978 in Dongcheng and Tongxian counties of Beijing and expanded to 161 points (each point corresponding to a county or district) in 31 provinces covering around 73 million population in 2004 [2]. The VR system was built in the 1950s in 13 cities and progressively enlarged to 319 counties/districts in 22 provinces covering about 230 million people in 2012 [3]. These two systems overlapped in 42 counties [3]. The VR system, while not representative, was able to provide more accurate estimates of the proportion of deaths due to specific causes because of the larger sample of deaths than the DSP system. Conversely, the DSP system reflected the total mortality, the broad causeof-death distribution, and the geographic distribution of deaths more accurately, because of the nationally representative sampling strategy. Differences between the two systems and their development are described in detail elsewhere [3].

In 2013, the VR and DSP systems were integrated and expanded to 605 points to become the new DSP system under the responsibility of China $\mathrm{CDC}$, covering approximately one quarter of the total population of China and providing representative death data at both national and provincial levels [3]. Since the late 1990s, many provincial CDCs expanded their own death surveillance and some counties also collected death data [4]. Meanwhile, starting in 2004, the vast majority of hospitals in China also reported deaths to the China CDC [5]. Collectively, the new DSP system, the expanded provincial and county (non-DSP) registration system, and the inhospital death reports form the China Cause of Death Reporting System (CDRS) report over 7.5 million deaths annually in real time using an internet-based reporting system established and operated by China CDC. Given the procedures and mandates of the three individual sources of mortality data, there is almost no duplication. Further, the death data from the CDRS is regularly verified and compared with data from the Ministry of Public
Security and the Ministry of Civil Affairs of China to create a single, comprehensive mortality surveillance system [6]. The China CDRS was established to capture every death in every county as mandated by the National Health and Family Planning Commission in 2014 [7]. In 2018 , the number of deaths registered by the CDRS was 7.7 million or about three quarters of the 10.5 million deaths estimated to have occurred in 2017 [8].

Although China has made tremendous progress in capturing deaths, approximately one in four deaths still go uncounted, with implications for reliably measuring the impact of programs designed to reduce mortality. Given the regional heterogeneity of key factors likely to affect death registration, including economy, health resource allocation, data collection policy enforcement, and coordination among multiple institutions [1, 9], death registration completeness can be expected to vary substantially across counties. However, the extent of this variation in completeness has never been assessed in China. Such analyses would not only provide the critical intelligence to monitor the performance of the death registration system, and to target interventions accordingly, but also provide the adjustment factors to correct death registration data to produce more reliable mortality statistics that serve the current needs of policymakers.

In this paper, we provide comprehensive estimates of death registration completeness for each of the 2844 Chinese counties in 2018 based on the CDRS. It is unlikely that targeted support to improve mortality surveillance systems in China can be effectively garnered from provincial-level analyses, given the heterogeneous performance of counties within provinces. We also assess how completeness is related predicted to likely key determinants, including economic status, availability and quality of healthcare, and population density.

\section{Methods \\ Data}

We used China CDRS as our source of death registration data. The CDRS in principle covers the entire population in each county or district. Death occurring in any county should be reported to the CDRS by that county, whether for local residents (i.e., persons living in the county for more than 6 months) or non-local residents. For each decedent, information is collected on basic demographic characteristics, residential address, place of death, and cause of death. Decedents are counted as local residents. Where they had lived in the county reporting the death less than 6 months, they are counted in their previous counties of residence. Resident population counts by county, age, and sex were obtained from the National Bureau of Statistics (NBS). The population at the end of 2017 was used as the mid-year population of 2018. Summary statistics on registered deaths and 
population size for each county (according to quartiles) are provided in Additional file 1: Table S1.

We also collated county-level data in 2017 on the gross domestic product (GDP) per capita, mean years of education per capita, and proportion of minority population from the national census and provincial-, city-, and county-level statistical reports. We evaluated the quality of these original data. Some data were missing for about $30 \%$ in some counties; further, we found that the values of GDP and mean education years in some counties for some years were very low or very high. To improve the reliability of these estimates, for GDP, we performed a spatiotemporal analysis to impute missing values and smooth the trend. For mean years of education, we used a proportional change model to project the annualized rate of change between 2000 and 2010 in order to obtain the value for 2017. We computed lag-distributed income (LDI) as the weighted average of GDP over a 5-year period. The average population density at the county level was estimated by dividing the resident population by county area (square kilometer) obtained from the $\mathrm{Na}-$ tional Ministry of Civil Affairs [10]. Additionally, scores on healthcare access and quality (HAQ) were obtained at the provincial level from the Global Burden of Disease 2017 (GBD2017) Study, reflecting health system capacity for timely and efficient detection, management, and treatment of disease [11]. The proportion of ethnic minority population was categorized into quartiles since only when the proportion of ethnic minority population in a region reaches a certain level will it be expected to clearly demonstrate an effect on death registration completeness.

\section{Estimation of completeness of death registration}

We estimated the death registration completeness of the CDRS by using the empirical completeness method developed by Adair and Lopez [12]. This method predicts completeness of death registration based on relatively limited data: registered crude death rate, under- 5 mortality rate $(5 \mathrm{q} 0)$, population age structure, and under- 5 death registration completeness. The method has significant advantages over traditional demographic methods to estimate completeness, particularly death distribution methods, in that it does not depend on stringent and often unrealistic assumptions about population stability and migration, can be readily applied at subnational levels, and produces results for recent years. More details about the model and coefficients used in this study are provided in Additional file 1: Table S2.

The GBD Study has estimated Chinese county-level $5 q 0$ for both sexes for 2012, the only study to our knowledge to do so [13]. The county-level 5q0 (and 95\% uncertainty interval [UI]) for 2018 was estimated by assuming that the ratio of county to province $5 \mathrm{q} 0$ for both sexes did not vary between 2012 and 2018 and the 2018 county-level ratio of sex-specific to both sexes $5 \mathrm{q} 0$ did not vary within each province (Additional file 1: $\mathrm{p}$ 2 ). Given the relatively brief time interval, and the already comparatively low levels of $5 \mathrm{q} 0$ in China in 2012, this assumption would appear reasonable.

At the national level, we also estimated completeness of death registration using the $5 \mathrm{q} 0$ from the United Nations Inter-Agency Group for Child Mortality Estimation (IGME) [14]. However, it should be noted that the model itself was developed using GBD 5q0 and all-age mortality data, including from China, and so to ensure consistency of the relationship between these variables and hence produce reliable estimates it is preferred to use the GBD rather than IGME $5 \mathrm{q} 0$.

We calculated 95\% UI for the completeness estimates by accounting for uncertainty arising from (1) the empirical completeness model, (2) 5q0 (95\% uncertainty intervals were provided by the GBD; we incorporated these assuming a normal distribution of the natural $\log$ of $5 \mathrm{q} 0$ ), and (3) stochasticity in the number of registered deaths (assuming a Poisson distribution). For each province and county, we produced 1000 simulations of completeness incorporating the three types of uncertainty simultaneously in each simulation. That is, in each simulation, the $5 \mathrm{q} 0$ and number of registered deaths were randomly generated based on their mean and assumed distribution; these were input into the model, which produced a completeness estimate that also incorporated the variance of each model coefficient. The $95 \%$ UI for completeness is then defined by the 2.5 and 97.5 percentiles of 1000 completeness estimates generated by the simulation results.

We investigated intra-provincial variation in completeness of death registration by computing the root mean square difference (RMSD) of county-level completeness compared with provincial-level completeness (Additional file 1: Table S4).

We undertook an independent sensitivity analysis to examine the robustness of the completeness estimation using $5 \mathrm{q} 0$ and population data from the separate death registration system for Anji, Yunhe, and Longyou Counties of Zhejiang province. These data cover the entire Hukou population (a population that is registered in the province) and is a different system to the CDRS system run by the China CDC. We used this data source to estimate completeness of death registration in these counties and compared them to the estimates in the primary analysis of this study. The Hukou death registration system of Zhejiang, a very developed province, is subject to strong data quality controls, and some counties (e.g., Anji, Yunhe, and Longyou Counties) have a very high proportion of the resident population that are registered as Hukou. Hence, the $5 \mathrm{q} 0$ and population data for these 
counties are likely to be valid and independent alternative inputs to estimate death registration completeness.

\section{Socio-economic determinants of completeness of death registration}

Using a two-level (provincial and county levels) logistic regression model (Additional file 1: p 3-p4), we explored the association between county-level completeness and a set of covariates reflecting socioeconomic status and healthcare quality, including income, education, minority population, population density, and healthcare access. We also considered whether the county was included in the DSP system, because this would likely led to greater awareness of the need to register deaths and more intensive surveillance procedures to do so. In particular, registration-enhancing policies and strategies were more likely to be implemented in the DSP counties, which can be expected to favorably influence the timeliness and completeness of death information reporting to the CDRS. A step-wise approach to model fitting was used; firstly, the covariates relating to the population were included in the model, followed by system-level covariates.

The median rate ratio (MRR) [15] was used to estimate geographic variation in completeness between two randomly selected provinces/counties. MRR is defined as the median value of the odds ratios (OR) between two random areas and calculated by translating area-level variance into an OR scale (Additional file 1: p 4). An MRR equal to 1 suggests no geographical variation in completeness, while MRR above 1 indicates that geographical variation is present. The contributions of these socioeconomic determinants can be assessed through the OR and its 95\% confidence interval (95\% CI) and the proportional change in variance (PCV) [16] (Additional file 1: p4) at the provincial and county levels.

We also used the variance inflation factor (VIF) function to assess whether there was multicollinearity among these socioeconomic determinants in the model. The VIF for each determinant (Additional file 1: Table S5) was much lower than 10, implying that these determinants were not correlated and could be applied in the model. SAS9.4 was used for all regression analyses.

\section{Results}

In 2018, the all-age completeness of death registration of the China CDRS was estimated using $5 \mathrm{q0}$ from the GBD to be $74.2 \%$ (95\% UI 66.2-80.4) for both sexes, with very little difference for males and females. Using the alternative, lower $5 \mathrm{q} 0$ from the IGME, implying much higher under-5 completeness, the all-age estimated completeness was much higher, at $83.0 \%$, 95\% UI 77.1-87.1 (see Additional file 1: Table S3). There was a wide geographical variation in death registration completeness among provinces, ranging from $15.6 \%$ (Tibet) to $94.3 \%$
(Liaoning). Provinces with higher under-5 completeness had higher all-age completeness, as expected. Apart from Liaoning, the highest estimated levels of completeness for all ages were reported for Jiangsu (93.0\%) and Fujian (89.8\%), while the lowest completeness occurs in the southwest and northwest provinces, including, in addition to Tibet, Xinjiang (30.4\%) and Guizhou (37.1\%).

Our findings show that death registration completeness for children under-5 was uniformly lower than that for all ages in all provinces except for Tibet, where both measures were very low. The ratio of all-age to under- 5 completeness ranged from 1.3 in Shanghai to 4.5 in Hebei, with under-5 completeness between 22 and $76 \%$ across provinces (Table 1). This was similar to the age pattern of the incompleteness of death registration reported elsewhere [1, 17-20], no doubt reflecting the tendency for poorer populations in particular to fail to register both the birth and the death when a child dies in the first few weeks or months of life.

As might be expected, the variation in death registration completeness was higher across counties than across provinces, reinforcing the policy value of assessing completeness at the county level. At the county level, the completeness ranged from 2.4\% (95\% UI 1.0-5.0\%) in Burang County, Tibet, to $100.0 \%$ (95\% UI 99.9-100.0\%) in Guandu District, Yunnan. More details on the countylevel completeness are provided in Additional file 1: Table S6 which shows, for each of the 2844 Chinese counties, the registered death rate and the estimated death registration completeness in 2018. Six hundred eighty (23.9\%) counties in China had estimated levels of completeness higher than $90 \%$, including 88 of 96 counties in Jiangsu (91.7\%), 65 of 100 counties in Liaoning (65.0\%), 78 of 122 counties in Guangdong (64.5\%), 44 of 83 counties in Fujian $(53.0 \%)$, and 8 of 16 counties in Shandong (50.0\%). These counties were concentrated mostly in the east and southeast coastal provinces with more developed economies (Fig. 1). By contrast, 197 (7.0\%) counties had estimated completeness lower than 20\%, mostly located in Tibet (52 of 74 counties), Guizhou (37 of 87 counties), Xinjiang (25 of 98 counties), and Gansu (14 of 87 counties).

Perhaps more surprisingly, several underdeveloped provinces, including Guangxi, Guizhou, Sichuan, Qinghai, and Inner Mongolia, with lower levels of overall completeness (Table 1), contained several counties where completeness was relatively high (Fig. 1). For example, in Guizhou, although death registration completeness was less than $30 \%$ in nearly half the counties, 11 of 87 counties had completeness higher than $85 \%$, including Kaiyang County (97.0\%, 95\% UI 93.5-99.0), Nanming District (95.3\%, 89.1-98.4), and Jiangkou County (95.2\%, 88.2-98.7), among others. In the coastal provinces of Jiangsu, Guangdong, and Fujian with high 
Table 1 Completeness of death registration at all ages and age under 5 years, by province, China, 2018

\begin{tabular}{|c|c|c|c|c|c|c|}
\hline \multirow[t]{2}{*}{ Province } & \multicolumn{3}{|c|}{ Death registration completeness (\%), all ages } & \multicolumn{3}{|c|}{ Death registration completeness (\%), under 5 years } \\
\hline & Both sexes & Male & Female & Both sexes & Male & Female \\
\hline China & $74.2(66.2,80.4)$ & $73.6(65.9,80.4)$ & $75.1(67.5,81.2)$ & 30.6 & 30.7 & 30.6 \\
\hline Liaoning & $94.3(91.4,96.5)$ & $93.9(90.7,95.9)$ & $94.0(90.8,96.2)$ & 59.6 & 58.9 & 61.0 \\
\hline Jiangsu & $93.0(88.7,95.6)$ & $91.5(87.4,94.4)$ & $93.5(89.9,96.0)$ & 60.6 & 59.3 & 62.4 \\
\hline Fujian & $89.8(84.4,93.3)$ & $89.5(84.9,93.4)$ & $89.8(84.6,93.1)$ & 51.6 & 53.3 & 49.0 \\
\hline Shanghai & $89.5(84.4,93.8)$ & $88.2(82.8,92.2)$ & $89.6(84.6,93.7)$ & 69.2 & 68.8 & 70.0 \\
\hline Guangdong & $86.7(80.2,91.3)$ & $85.7(79.1,90.1)$ & $87.6(81.6,92.3)$ & 60.7 & 61.3 & 59.5 \\
\hline Yunnan & $84.2(76.1,90.3)$ & $84.4(75.7,90.7)$ & $82.8(75.3,88.6)$ & 52.8 & 55.7 & 49.1 \\
\hline Zhejiang & $83.4(76.6,88.4)$ & $82.3(75.9,87.5)$ & $84.4(78.2,89.6)$ & 40.7 & 40.4 & 41.0 \\
\hline Shandong & $83.1(76.7,88.6)$ & $82.4(75.6,87.9)$ & $83.3(76.5,88.6)$ & 34.9 & 34.1 & 35.9 \\
\hline Hunan & $82.9(76.4,88.3)$ & $82.0(75.2,87.1)$ & $84.0(77.5,89.1)$ & 32.6 & 32.2 & 33.1 \\
\hline Tianjin & $82.8(76.2,87.9)$ & $78.5(71.2,84.6)$ & $87.4(82.1,91.2)$ & 39.4 & 37.5 & 42.1 \\
\hline Beijing & $80.7(73.0,87.7)$ & $79.5(72.2,85.8)$ & $82.9(75.5,89.0)$ & 49.4 & 49.1 & 50.5 \\
\hline Henan & $76.8(67.1,84.2)$ & $75.7(66.5,83.3)$ & $79.7(71.7,86.4)$ & 23.5 & 22.2 & 25.4 \\
\hline Chongqing & $76.7(68.9,83.7)$ & $75.9(67.6,82.2)$ & $76.7(68.4,83.5)$ & 24.4 & 24.0 & 25.1 \\
\hline Jilin & $76.3(68.1,82.6)$ & $77.6(69.6,84.0)$ & $75.1(66.4,82.2)$ & 33.3 & 34.7 & 31.5 \\
\hline Hubei & $75.0(65.6,82.3)$ & $75.2(67.3,81.6)$ & $74.3(65.1,81.2)$ & 33.0 & 33.8 & 31.7 \\
\hline Ningxia & $74.6(64.5,82.4)$ & $72.5(61.5,81.5)$ & $75.8(67.8,83.8)$ & 48.4 & 46.8 & 50.7 \\
\hline Guangxi & $73.6(65.8,80.9)$ & $77.1(69.5,82.8)$ & $70.0(61.5,76.8)$ & 30.2 & 32.7 & 26.8 \\
\hline Heilongjiang & $72.4(63.7,79.2)$ & $72.9(64.8,79.6)$ & $71.9(63.0,79.4)$ & 24.7 & 23.2 & 27.0 \\
\hline Anhui & $70.3(61.6,77.5)$ & $71.3(63.0,78.4)$ & $69.5(59.8,77.4)$ & 23.1 & 25.1 & 20.2 \\
\hline Sichuan & $69.5(59.6,77.6)$ & $68.9(59.0,77.9)$ & $67.8(58.1,75.9)$ & 27.4 & 28.3 & 26.3 \\
\hline Hebei & $68.0(59.1,75.8)$ & $67.9(59.1,76.1)$ & $70.6(61.2,78.3)$ & 15.1 & 14.8 & 15.5 \\
\hline Inner Mongolia & $66.0(56.0,74.4)$ & $65.8(56.1,74.0)$ & $66.5(57.1,75.5)$ & 28.6 & 27.0 & 31.2 \\
\hline Shanxi & $61.6(52.3,69.7)$ & $59.9(50.6,68.7)$ & $66.5(57.6,74.5)$ & 21.4 & 20.6 & 23.0 \\
\hline Jiangxi & $60.4(50.3,68.4)$ & $59.7(50.3,67.9)$ & $61.2(52.3,69.9)$ & 24.9 & 25.2 & 24.4 \\
\hline Qinghai & $59.7(48.6,71.1)$ & $58.8(46.4,70.2)$ & $60.9(49.8,70.0)$ & 41.8 & 43.0 & 40.6 \\
\hline Shaanxi & $57.3(45.4,65.4)$ & $56.2(45.6,67.5)$ & $57.4(47.7,67.8)$ & 15.4 & 15.8 & 14.8 \\
\hline Gansu & $43.3(34.8,52.3)$ & $42.4(33.3,51.1)$ & $46.7(37.5,55.7)$ & 16.0 & 15.5 & 16.8 \\
\hline Hainan & $41.0(32.8,50.2)$ & $47.6(38.2,57.1)$ & $38.1(29.8,48.4)$ & 17.3 & 18.4 & 16.2 \\
\hline Guizhou & $37.1(27.8,47.5)$ & $39.8(29.4,51.6)$ & $35.3(26.6,44.2)$ & 23.4 & 24.1 & 22.3 \\
\hline Xinjiang & $30.4(22.5,39.9)$ & $30.0(20.8,39.5)$ & $34.8(25.6,43.9)$ & 19.2 & 19.6 & 19.1 \\
\hline Tibet & $15.6(10.7,22.8)$ & $15.1(10.5,21.6)$ & $18.8(13.3,25.9)$ & 15.7 & 17.5 & 13.6 \\
\hline
\end{tabular}

levels of overall completeness, there were nonetheless still a few counties with low completeness, such as Qinghe District $(44.9 \%, 29.0-63.8)$ in Jiangsu, Suixi County $(47.6 \%, 35.9-61.5)$ in Guangdong, and Fu'an City $(27.3 \%, 19.9-35.9)$ in Fujian. These observations are perhaps counterintuitive but point to the influence of strong local factors which appear to affect levels of under-registration in unpredictable ways but which need to be understood if overall death registration in China is to improve significantly on current levels. The findings also confirmed the policy utility of assessing completeness levels for as local a population as possible in order to more effectively target intervention strategies.

Table 2 presents the results of the sensitivity analysis. The population counts and age structure (the fraction of the population aged 65 years old and over) of Anji, Yunhe, and Longyou Counties were taken from the NBS, and the counties were very close. Due to the slightly higher $5 \mathrm{q} 0$ from the counties than the GBD (and therefore lower under-5 completeness), the all-age estimated completeness of death registration was slightly 


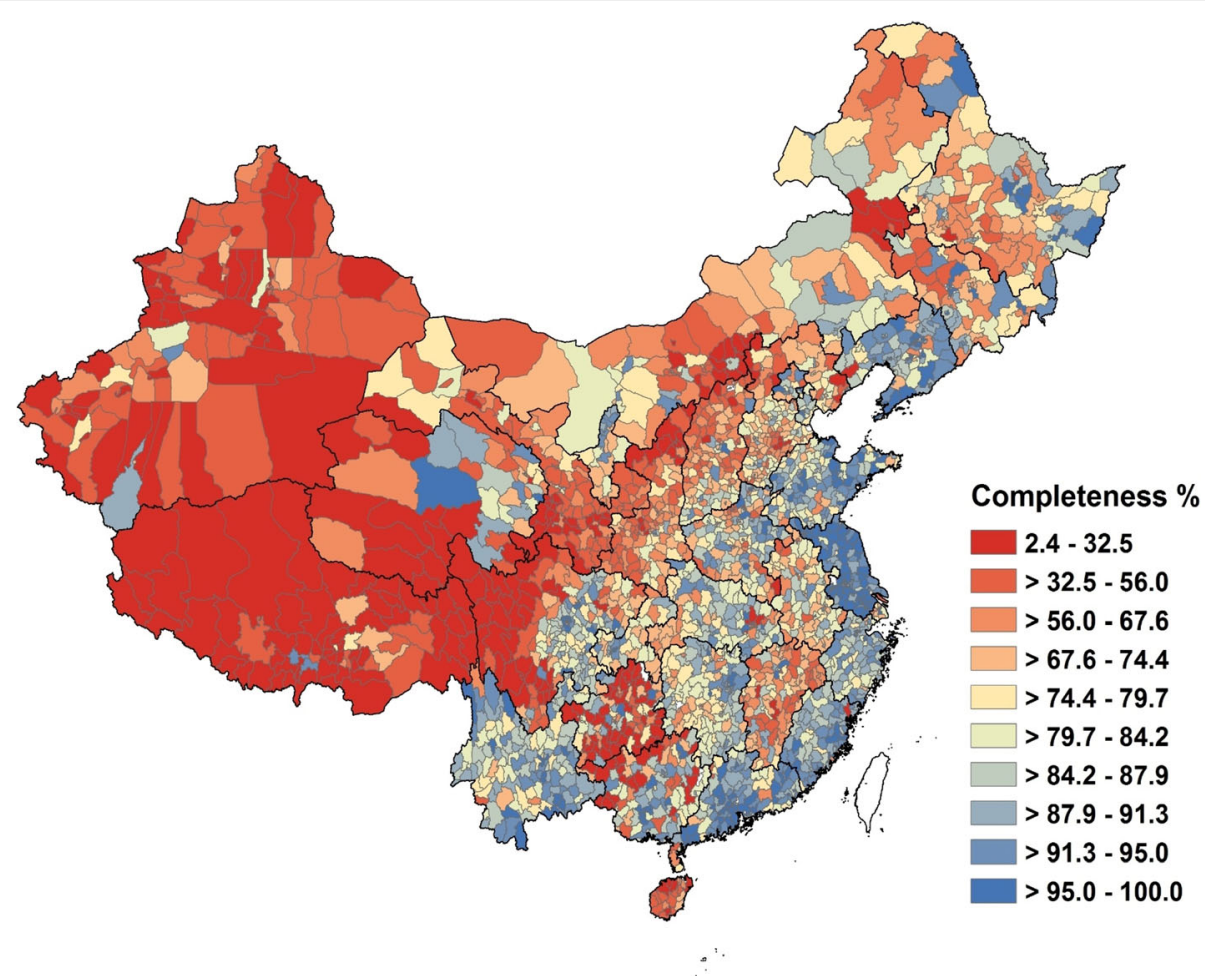

Fig. 1 Death registration completeness (\%) for both sexes in 2844 Chinese counties in 2018

lower (less than two percentage points difference) when we used the $5 \mathrm{q} 0$ and population data from the county as input data. However, these differences were small. The consistent findings from the application of alternative data sources provide some support for the robustness of the empirical completeness model outputs.
To assess how death registration completeness varied with possible determinants, we provide scatter plots to visualize the relationship between completeness and some potential key socioeconomic determinants (Fig. 2). Of these, mean education years per capita (Fig. 2a), lagdistributed income (Fig. 2b), and average population

Table 2 Completeness of death registration in Anji, Yunhe, and Longyou Counties of Zhejiang province: sensitivity analysis based on different data sources of $5 \mathrm{q} 0$ and population structure

\begin{tabular}{|c|c|c|c|}
\hline & Anji County & Yunhe County & Longyou County \\
\hline Registered deaths from the CDRS & 3213 & 837 & 2776 \\
\hline $5 q 0$ from the GBD study $(1 / 1000)$ & 5.5 & 6.7 & 7.5 \\
\hline $5 q 0$ from the county $(1 / 1000)$ & 5.9 & 7.5 & 8.2 \\
\hline Population from the National Bureau of Statistics (NBS) & 484,902 & 114,700 & 370,799 \\
\hline$\% 65$ years old and over from the NBS (\%) & 13.1 & 12.9 & 15.4 \\
\hline Population from the county & 477,337 & 113,954 & 385,879 \\
\hline$\% 65$ years old and over from the county (\%) & 14.3 & 13.3 & 15.9 \\
\hline Death registration completeness $(\%)^{a}$, under 5 years & 42.1 & 12.2 & 70.1 \\
\hline Death registration completeness $(\%)^{a}$, all ages & 89.9 & 82.8 & 91.8 \\
\hline Death registration completeness $(\%)^{\mathrm{b}}$, under 5 years & 40.6 & 11.4 & 67.9 \\
\hline Death registration completeness $(\%)^{\mathrm{b}}$, all ages & 88.2 & 81.7 & 88.9 \\
\hline
\end{tabular}

${ }^{\mathrm{a}}$ Death registration completeness was estimated using the $5 \mathrm{q} 0$ of the GBD and population from the NBS

${ }^{\mathrm{b}}$ Death registration completeness was estimated using the $5 \mathrm{q} 0$ and population from the counties 


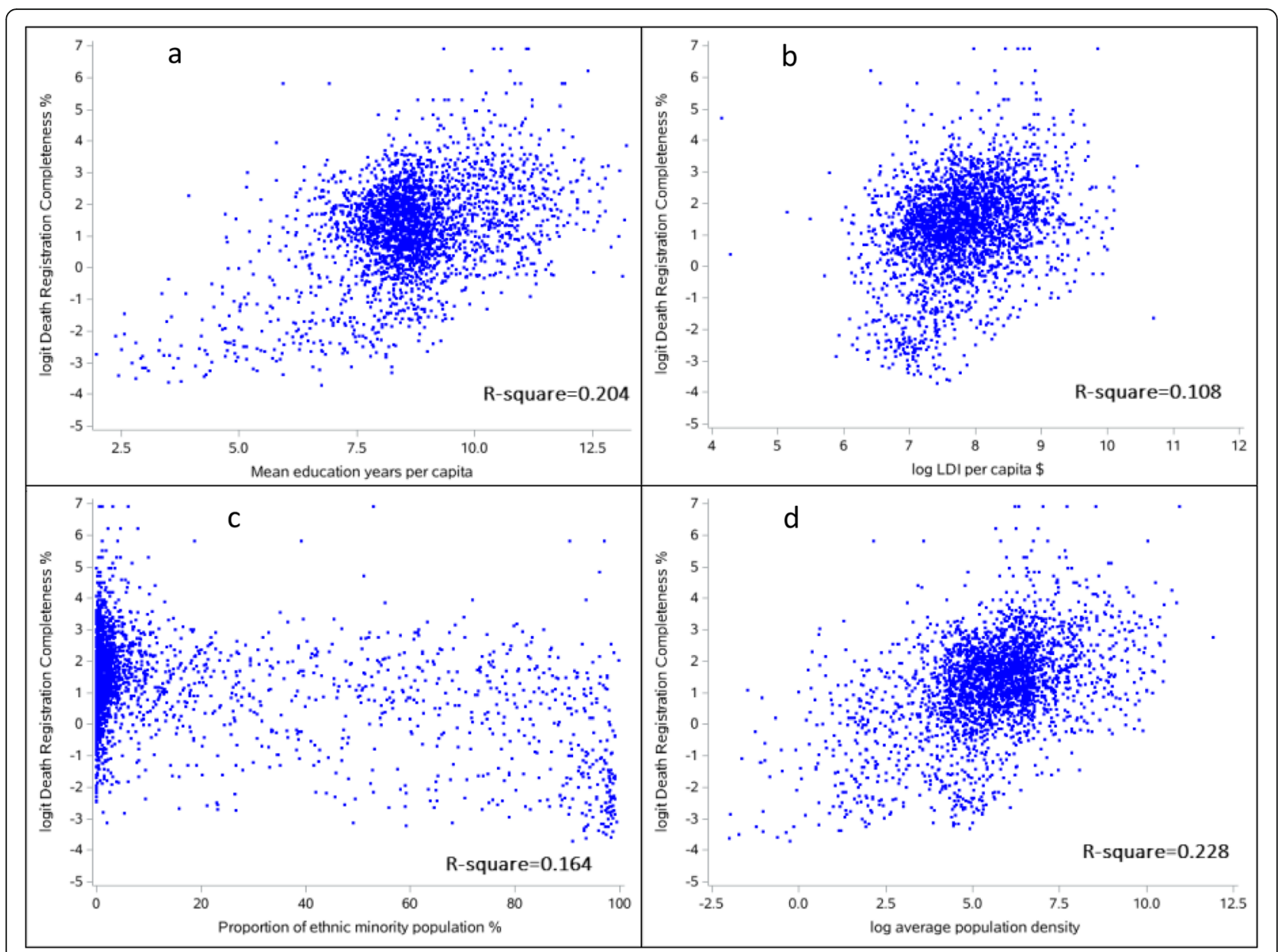

Fig. 2 Scatter plots for death registration completeness (\%) and socioeconomic determinants for both sexes in 2844 Chinese counties in 2018

density (Fig. 2d) were weakly positively related to levels of completeness ( $R^{2}$ respectively of $0.20,0.11$, and 0.23 ). Conversely, the higher proportion of ethnic minority in the population (Fig. $2 \mathrm{c}, R^{2}=0.16$ ), the lower the level of completeness, although again, the association was comparatively weak.

We ascertained what might be the main drivers of these observed geographical differences in completeness of death registration by fitting a two-level (province and county) logistic regression model (Table 3). Mean education years, income, and population density were all positively associated with completeness. On the other hand, the regions which had a high proportion of minority ethnicities in the population tended to have lower completeness. There was no statistically significant difference in completeness between males and females (model 1).

After the inclusion of a variable signifying whether the county is a DSP or not, as well as the HAQ Index for each province, PCV for the province level significantly increased, implying better health services were potentially important for promoting death registration completeness, as estimated in model 2. Compared to DSP counties, non-DSP counties had around $40 \%$ lower completeness. HAQ Index was positivity associated with completeness. Overall adjustment for all socioeconomic determinants accounted for approximately $53.1 \%$ of the province-level and $16.7 \%$ of the county-level variation in death registration completeness.

\section{Discussion}

In this study, we assess death registration completeness at both the province and county levels in China and corresponding determinants. These results will be important for central and local governments to target further efforts to strengthen death registration in areas with markedly lower completeness. The results will also aid researchers and public health workers to more reliably adjust deficient death registration data on a county-bycounty basis to produce more accurate mortality statistics at the county, provincial, and national levels.

Our findings have immediate relevance for the monitoring of progress with national development goals and 
Table 3 Socio-economic determinants of completeness of death registration (logit) in China in 2018

\begin{tabular}{|c|c|c|c|c|}
\hline & \multicolumn{2}{|l|}{ Model 1} & \multicolumn{2}{|l|}{ Model 2} \\
\hline & OR $(95 \% \mathrm{Cl})$ & $P$ & $\mathrm{OR}(95 \% \mathrm{Cl})$ & $P$ \\
\hline \multicolumn{5}{|l|}{ Fixed effects } \\
\hline Gender (ref: male) & 1 & & 1 & \\
\hline Female & $1.05(0.99,1.11)$ & 0.10 & $1.05(0.99,1.11)$ & 0.09 \\
\hline Mean education years per capita & $1.31(1.27,1.36)$ & $<0.01$ & $1.30(1.26,1.35)$ & $<0.01$ \\
\hline Log lag distribute income per capital (\$) & $1.14(1.08,1.21)$ & $<0.01$ & $1.13(1.07,1.19)$ & $<0.01$ \\
\hline Log population density (person per $\mathrm{km}^{2}$ ) & $1.04(1.01,1.07)$ & $<0.01$ & $1.03(1.01,1.06)$ & 0.02 \\
\hline Proportion of ethnic minority population (\%) (ref: 0-0.3) & 1 & & 1 & \\
\hline $0.3-1.5$ & $0.96(0.89,1.05)$ & 0.37 & $0.96(0.89,1.04)$ & 0.34 \\
\hline $1.5-13.1$ & $0.92(0.84,1.01)$ & 0.09 & $0.91(0.83,1.01)$ & 0.07 \\
\hline $13.1-99.8$ & $0.66(0.59,0.75)$ & $<0.01$ & $0.66(0.59,0.74)$ & $<0.01$ \\
\hline DSP (ref: yes) & & & 1 & \\
\hline No & & & $0.60(0.57,0.64)$ & $<0.01$ \\
\hline HAQ Index & & & $1.04(1.01,1.07)$ & 0.01 \\
\hline \multicolumn{5}{|l|}{ Random effects } \\
\hline Province-level variance (SE) & $0.617(0.162)$ & & $0.519(0.139)$ & \\
\hline MRR (provinces) & 2.12 & & 1.99 & \\
\hline PCV (provinces) (\%) & 44.14 & & 53.05 & \\
\hline County-level variance (SE) & $1.101(0.021)$ & & $1.059(0.02)$ & \\
\hline MRR (counties) & 2.72 & & 2.67 & \\
\hline PCV (counties) (\%) & 13.39 & & 16.68 & \\
\hline
\end{tabular}

improving understanding of health and mortality inequalities in China. As a response to the United Nations Sustainable Development Goals, the Chinese government in 2016 issued Healthy China 2030 in which they proposed explicit mortality-related targets, such as by 2030, increasing life expectancy to 79.0 years reducing infant and under-5 mortality to $5.0 \%$ and $6.0 \%$, respectively; and decreasing maternal mortality to 12 per 100 , 000 people, and requested each province to set their own targets [21, 22]. Timely and complete death registration data covering the whole population is fundamental for quantifying and monitoring progress on these indicators. We estimate that death registration completeness of the Chinese CDRS was $74.2 \%$ in 2018, very similar to that (73.6\%) estimated by the GBD study 2017 using quite different methods and data sources [8]. A previous study [23] had reported that the estimated death registration completeness in the CDRS was 56\% (6.0 million registered deaths) in 2014 and 58\% (6.2 million registered deaths) in 2015 using capture-recapture methods. Over the past 5 years, there has been a tremendous improvement in the coverage and completeness of the CDRS, with the annual number of deaths registered increasing from 6.0 million in 2014 to 7.7 million in 2018. Some studies [24-26] of mortality data reported in China's Population Census Data have shown serious under-reporting of deaths, especially among children and the population aged 60 years and over. For example, in the 2010 census, the completeness of death registration was $40 \%$ and $80 \%$ respectively for children and the elderly population [26]. Death registration completeness based on the DSP system has also been reported in previous studies $[19,27]$ noting that completeness was generally higher in the DSP populations due to the implementation of registration-enhancing policies and strategies.

There are significant geographical differences in death registration completeness across counties in China. The coastal and wealthier regions in eastern and southeastern China generally have higher completeness than those in the west and central regions, although somewhat surprisingly, these provinces with high levels of overall completeness contained several counties with very low completeness, such as Jianchang County in Liaoning, Qinghe District in Jiangsu, Fu'an City in Fujian, and Suixi County in Guangdong. Although it is important for central and provincial governments to provide leadership and promote certain policies and strategies on death registration [28], how these are implemented varies considerably at the county level.

The National Health and Family Planning Commission, the Ministry of Public Security, and the Ministry of 
Civil Affairs of China have jointly issued guidelines to strengthen the administration of death registration, emphasizing the need for data verification and exchange of information among the three departments [6]. As a result, many regions have strengthened procedures for sharing data on deaths across multiple sectors $[29,30]$. Not all have, however, due to the non-mandatory nature of the guidance and the lack of attention paid by local governments to death surveillance. In addition to further promoting multi-sectoral data exchange, there will be scope for greater triangulation of existing data sources in order to better understand data quality and biases. For example, a systematic program of data comparison and verification between the CDRS and the Population Registration Database maintained by the Center for Health Statistics and Information of the National Health Commission in China [22] would yield immediate benefits in terms of improving understanding of data quality and factors affecting completeness.

Interestingly, in some underdeveloped and remote provinces with low levels of overall death registration completeness, such as Guangxi, Guizhou, Sichuan, Qinghai, and Inner Mongolia, there are counties which have very high completeness. For example, within Guizhou, completeness in Kaiyang County, Nanming District, Qingzhen City, and so on exceeded $90 \%$. All of these counties were included in the DSP system where multiple specific strategies are used to promote death registration, including special funding by the central government, dedicated staff in hospitals and CDCs who are responsible for registering deaths, regular training and supervision of staff by national and provincial CDCs, critically reviewing death data annually for completeness and accuracy, conducting periodic surveys of deaths under-reporting, and disseminating statistical reports on mortality to the public to raise awareness [2, 3]. Conversely, non-DSPs counties receive much less support from governments and relevant departments and have less funding and staff. Our study confirms that inclusion in the DSP system, with all of its associated datapromoting advantages, was an important determinant of death registration completeness at the county level, particularly in poorer provinces. This factor alone contributed substantially to increasing completeness among counties in some provinces.

Our study has suggested that more minority populations, dispersed population distribution, and lower quality of healthcare were all important factors associated with lower death registration completeness. The majority of counties in Tibet and Xinjiang have very low completeness, and $91 \%$ and $63 \%$ of the total population in Tibet and Xinjiang, respectively, were minorities [31]. Given their own special culture, beliefs, and customs, most people choose to die at home [32, 33]. After death, they are generally not cremated, but instead undergo a "celestial" burial (corpses eaten by vultures) or are inhumed [34], neither of which requires a death certificate. Furthermore, Tibet and Xinjiang are both vast and sparsely populated regions [9]. In agriculture areas and remote villages, difficult natural environmental conditions and inconvenient transportation present serious barriers for family members to report the home deaths and for village doctors to conduct surveys of underreporting. In 2015, health system performance in Tibet and Xinjiang was very poor [10]. Inadequate access to healthcare also undoubtedly contributes to the low registration completeness in these regions. In addition, local governments do not provide funds to support death registration, and mechanisms for data exchange across multiple sectors have not been established.

It is reasonable to expect that higher-level socioeconomic development might contribute substantially to death registration completeness, given the experience of more developed countries where very few deaths go unrecorded [35]. Yet, our results indicated that developmental factors such as income, educational attainment, and population density only account for a low proportion of the geographical variation across China. One might have expected a more substantial contribution from this amalgam of development factors, suggesting that more research is needed to more precisely delineate the impact of socioeconomic development, and how it is being modulated, at local and/or national level, on death registration completeness. The influence of socioeconomic conditions is clearly important and seemingly poorly understood, likely affected by staffing, policy implementation, quality of medical services, access to healthcare, health behavior, and awareness of the public about the importance of registering births and deaths, among others. Indeed, they may well play a greater role in raising levels of death registration completeness than classical measures of development such as education and wealth. It would appear that while socioeconomic development is a major underlying determinant of health policies, strategies, and actions, including death registration, once a certain level of socioeconomic development has been achieved, other factors related to the strength of commitment to improving data for policy may well influence death registration much more.

\section{Limitations}

This first-ever comprehensive estimation of death registration completeness based on the China CDRS at the provincial and county levels has some limitations. First, the empirical completeness model was developed using GBD data, which are themselves based on demographic and statistical models. However, the method's validity has been demonstrated elsewhere by its high 
concordance with completeness estimates in national populations not included in the database to develop the model, as well as subnational populations [12]. Second, the $5 \mathrm{q} 0$ for each county and province was a modelbased estimate taken from the GBD 2017 since this was the most recent and comprehensive source of these input data. We have incorporated the uncertainty around these estimates of $5 \mathrm{q} 0$ into our own uncertainty intervals of completeness. Third, due to China not having a fully functional vital registration system, the resident population counts by county have been taken from the NBS. These are projected populations, which could be another source of uncertainty for the completeness estimates. We conducted a sensitivity analysis using independent $5 \mathrm{q0}$ and population counts for three counties of Zhejiang province which maintain their own independent vital registration system to estimate the corresponding death registration completeness in the CDRS.

\section{Conclusion}

While China has been very successful in improving levels of death registration among its nearly 1.4 billion citizens, there are marked inequalities in registration completeness across the country. Overall, completeness is higher in more industrialized, wealthier eastern regions than in more rural, poorer western regions, but regional inequality is actually more complicated with significant variations in completeness across counties within both richer and poorer provinces. Socioeconomic condition, a faciliatory environment such as afforded by the presence of the DSPs, and access to quality medical care were the key drivers of the observed geographical variation in completeness in China. To improve death registration completeness further will require targeted research about how non-developmental factors impede or promote registration in those counties and provinces where levels are low. Doing so will ensure that health and social development policy across China will benefit from less biased information about who is dying prematurely, and where.

\section{Supplementary information}

Supplementary information accompanies this paper at https://doi.org/10. 1186/s12916-020-01632-8.

Additional file 1. Online material providing details of empirical completeness method, estimation of the county-level under-5 mortality rate for 2018, model for estimating association between county-level death registration completeness and socio-economic determinants, interprovincial variation in completeness of death registration and supplemental tables (Table S1-S6).

\section{Abbreviations}

5q0: Under-5 mortality rate; CDC: Centers for Disease Control and Prevention; CDRS: Cause of Death Reporting System; Cl: Confidence interval; DSP: Disease Surveillance Points; GBD: Global Burden of Disease;
HAQ: Healthcare access and quality; IGME: Inter-agency Group for Child Mortality Estimation; LDI: Lag-distributed income; MRR: Median rate ratio; OR: Odds ratio; PCV: Proportional change in variance; RMSD: Root mean square difference; UI: Uncertainty interval; VIF: Variance inflation factor

\section{Acknowledgements}

We thank all staff from the China Cause of Death Reporting System who have made a great contribution to the data collection, auditing, and database management.

\section{Authors' contributions}

PY, ADL, and MGZ conceived the study concept. XYZ and TA performed the data analysis. $X Y Z$ drafted the manuscript. TA, PY, and $A D L$ reviewed the manuscript and provided critical feedback. XYZ, LJW, PY, and MGZ take responsibility for the integrity of the data and the accuracy of the data analysis. JLQ, YNL, and JML reviewed the findings and contributed to the interpretation. All authors agreed with the final version of the paper.

\section{Authors' information}

Chinese Center for Disease Control and Prevention, Beijing, China (XY Zeng, $\mathrm{MPH})$; Melbourne School of Population and Global Health, The University of Melbourne, Carlton, Victoria, Australia (T Adair, PhD; Prof A D Lopez, PhD); National Center for Chronic and Noncommunicable Disease Control and Prevention, Chinese Center for Disease Control and Prevention, Beijing, China ( $\amalg J$ Wang, MPH; P Yin, PhD; JL Qi, PhD; YN Liu, MPH; JM Liu, MPH; Prof MG Zhou, PhD)

\section{Funding}

This research was supported by the China National Key Research and Development Program (grant number 2018YFC1315301). The funder had no role in the study design, data collection, data analysis, data interpretation, or writing of the manuscript.

\section{Availability of data and materials}

The datasets used in the current study are available from the corresponding author on reasonable request.

\section{Ethics approval and consent to participate}

As all data was summarized from the China CDRS; the consent to publication is not applicable. The ethics committee at the Chinese Center for Disease Control and Prevention approved the study.

\section{Consent for publication}

No individual data is presented, and consent to publication is therefore not applicable.

\section{Competing interests}

$\mathrm{ADL}$ is a Board Member for this Journal. Other authors have declared that they have no competing interests.

\section{Author details}

${ }^{1}$ Chinese Center for Disease Control and Prevention, Beijing, China. ${ }^{2}$ Melbourne School of Population and Global Health, The University of Melbourne, Carlton, Victoria, Australia. ${ }^{3}$ National Center for Chronic and Noncommunicable Disease Control and Prevention, Chinese Center for Disease Control and Prevention, 27\# Nanwei Road, Xicheng District, Beijing 100050, China.

Received: 8 December 2019 Accepted: 14 May 2020

Published online: 03 July 2020

\section{References}

1. Lopez AD, Mikkelsen L, Rampatige R, Upham S, AbouZahr C, Gamage S, de Savigny D, Schmider A, Strengthening civil registration and vital statistics for births, deaths and causes of death: resource kit. Geneva: World Health Organization; 2012.

2. Yang G, Hu JP, Rao KQ, Ma JM, Rao C, Lopez AD. Mortality registration and surveillance in China: history, current situation and challenges. Popul Health Metrics. 2005;3(1):3. 
3. Liu SW, Wu XL, Lopez AD, Wang LJ, Cai Y, Page A, et al. An integrated national mortality surveillance system for death registration and mortality surveillance, China. Bull World Health Organ. 2016;94(1):46-57.

4. Yang G. Selection of DSP points in second stage and their presentation. Chin J Epidemiol. 1992;13(4):197-201.

5. Zhou MG, Jiang Y, Huang ZJ, Wu F. Adjustment and representativeness evaluation of national disease surveillance points system. Dis Surveill. 2010; 25(3):239-44.

6. The National Health and Family Planning Commission, the Ministry of Public Security, the Ministry of Civil Affairs of the People's Republic of China. Notice to strengthen the administration of medical certificates and the registration of deaths. Bull Minist Public Secur People's Republic of China. 2014:1:19-26

7. The National Health and Family Planning Commission. Notice of the management of population death information registration. Bull Natl Health Family Plann Comm People's Republic of China. 2014;12:37-40.

8. GBD2017 Causes of Death Collaborators. Global, regional, and national agesex-specific mortality for 282 causes of death in 195 countries and territories, 1980-2017: a systematic analysis for the Global Burden of Disease Study 2017. Lancet. 2018;392(10159):1736-88.

9. AbouZahr C, Savigny D, Mikkelsen L, Setel PW, Lozano R, Nichols E, et al. Civil registration and vital statistics: progress in the data revolution for counting and accountability. Lancet. 2015;386(10001):1373-85.

10. National administrative division information inquiry: Ministry of Civil Affairs of the People's Republic of China. Available from: http://bmfw.www.gov.cn/ mzbqgxzghxxcx/index.html.

11. Zeng $X Y$, Wang $L$, Yin P, Liu YN, Liu JM, You JL, et al. Subnational analysis of healthcare access and quality in China during 1990-2015. Chin Sci Bull. 2018:63(25):2631-40.

12. Adair T, Lopez AD. Estimating the completeness of death registration: an empirical method. PLoS One. 2018;13(5):e0197047.

13. Wang YP, Li XH, Zhou MG, Luo SS, Liang J, Liddell CA, et al. Under-5 mortality in 2851 Chinese counties, 1996-2012: a subnational assessment of achieving MDG 4 goals in China. Lancet. 2016;387(10015):273-83.

14. UN Inter-agency Group for Child Mortality Estimation. Available from: https://childmortality.org/data/China.

15. Merlo J, Chaix B, Ohlsson H, Beckman A, Johnell K, Hjerpe P, et al. A brief conceptual tutorial of multilevel analysis in social epidemiology: using measures of clustering in multilevel logistic regression to investigate contextual phenomena. J Epidemiol Community Health. 2006;60(4):290-7.

16. Merlo J, Yang M, Chaix B, Lynch J, Rastam L. A brief conceptual tutorial on multilevel analysis in social epidemiology: investigating contextual phenomena in different groups of people. J Epidemiol Community Health. 2005;59(9):729-36.

17. Hong TT, Phuong Hoa N, Walker SM, Hill PS, Rao C. Completeness and reliability of mortality data in Viet Nam: implications for the national routine health management information system. PLoS One. 2018;13(1):e0190755.

18. Mahapatra P, Chalapati Rao PV. Cause of death reporting systems in India: a performance analysis. Natl Med J India. 2001;14(3):154-62.

19. Wang L, Wang LJ, Cai Y, Ma LM, Zhou MG. Analysis of under-reporting of mortality surveillance from 2006 to 2008 in China. Chin J Prev Med. 2011; 45(12):1061-4.

20. Prasartkul $P$, Vapattanawong $P$. The completeness of death registration in Thailand: evidence from demographic surveillance system of the Kanchanaburi Project. World Health Popul. 2006:8(3):43-51.

21. China life expectancy of 79 in 2030 health plan: the State Council of the People's Republic of China. Available from: http://english.www.gov.cn/ policies/latest_releases/2016/10/25/content_281475475062678.htm.

22. The "Healthy China 2030" blueprint: the Communist Party of China Central Committee and the State Council. Available from: http://www.gov.cn/ xinwen/2016-10/25/content_5124174.htm.

23. Yang RK, Xue M, Lin QS, Zhou XH, Cai Y. The methodology study of realizing the full coverage of vital registration in China through multi-source data comparison. Chin J Health Inform Manage. 2019;16(2):192-7.

24. Wang JY. Trends in life expectancies and mortality patterns in China since 1990: a further examination and analysis. Popul Res. 2013;37(4):3-18.

25. Li SZ, Sun FB. Mortality analysis of China's 2000 population census data: a preliminary examination. China Rev. 2003;3(2):31-48.

26. Wang JY, Ge YX. Assessment of 2010 census data quality and past population changes. Popul Res. 2013;37(1):22-33.
27. Guo K, Yin P, Wang LJ, Ji YB, Li QF, Bishai D, Liu SW, et al. Propensity score weighting for addressing under-reporting in mortality surveillance: a proofof-concept study using the nationally representative mortality data in China. Popul Health Metrics. 2015;13:16.

28. Carla A, Lisa Grace SB, Lourdes H, Gulnara K, Lyaziza S. Strengthening civil registration and vital statistics in the Asia-Pacific region-learning from country experiences. Asia-Pac Popul J. 2016;29(1):39-73.

29. Notice of the General Office of the Municipal Government on the issuance of Information Comparisons for the Qualification Certification of Personnel Enjoying Social Insurance Benefits in Nanjing. General Office of Nanjing Municipal People's Government. Available from: http://www.nanjing.gov.cn/ zdgk/201810/t20181022573270.html.

30. Notice of Beijing Municipal Health and Family Planning Commission on Further Standardizing the Management of Medical Certificates and Information Registration for Population Death: Beijing Municipal Health Committee. Available from: http://www.beijing.gov.cn/zfxxgk/110088/ yzygc23/2015-11/02/content_630592.shtml.

31. 2010 Population Census: National Bureau of Statistics of China. Available from: http://www.stats.gov.cn/english/Statisticaldata/CensusData/rkpc2010/ indexch.htm.

32. Zhou MG, Yang G. Factors associated with place of death in China. Dis Surveill. 2009:24(5):382-5.

33. Pingcuo ZM, Qi JL, Gama CJ, Li YJ. Analysis of major causes of death and burden of disease of residents in Tibet Autonomous Region in 2015. Dis Surveill. 2019;1(34):66-9.

34. Chen GB. A brief analysis of the impact of geographical environment on the changes of Tibetan funeral forms. History Issue. 2012;10:28-9.

35. Phillips DE, AbouZahr C, Lopez AD, Mikkelsen L, de Savigny D, Lozano R, et al. Are well functioning civil registration and vital statistics systems associated with better health outcomes? Lancet. 2015;386(10001):1386-94.

\section{Publisher's Note}

Springer Nature remains neutral with regard to jurisdictional claims in published maps and institutional affiliations.

Ready to submit your research? Choose BMC and benefit from

- fast, convenient online submission

- thorough peer review by experienced researchers in your field

- rapid publication on acceptance

- support for research data, including large and complex data types

- gold Open Access which fosters wider collaboration and increased citations

- maximum visibility for your research: over $100 \mathrm{M}$ website views per year

At BMC, research is always in progress.

Learn more biomedcentral.com/submissions 